\title{
The Fischer-Marsden Solutions on Almost CoKähler Manifold
}

\author{
Dhriti Sundar Patra* Arindam Bhattacharyya and Manjusha Tarafdar \\ (Communicated by Ramesh Sharma)
}

\begin{abstract}
In this paper, we characterize the solutions of the Fischer-Marsden equation $\mathcal{L}_{g}^{*}(\lambda)=0$ on an almost CoKähler manifold. We prove that the Fischer-Marsden equation has only trivial solution on almost CoKähler manifold of dimension greater than 3 with $\xi$ belonging to the $(\kappa, \mu)$-nullity distribution and $\kappa<0$.
\end{abstract}

Keywords: The Fischer-Marsden equation; almost CoKähler manifold; $(\kappa, \mu)$-almost CoKähler manifold; Einstein manifold

AMS Subject Classification (2010): Primary: 53D15 ; Secondary: 53C25; 53C21.

\section{Introduction}

Let $\mathcal{M}$ denote the set of Riemannian metrics on a compact orientable manifold $\left(M^{n}, g\right)$ of unit volume and $g$ be a Riemannian metric on $M^{n}$. Let $g^{*}$ be any symmetric bilinear form on $M^{n}$. Then linearization of the scalar curvature $\mathcal{L}_{g}\left(g^{*}\right)$ is given by

$$
\mathcal{L}_{g}\left(g^{*}\right)=-\Delta_{g}\left(\operatorname{tr}_{g} g^{*}\right)+\operatorname{div}\left(\operatorname{div}\left(g^{*}\right)\right)-g\left(g^{*}, R i c_{g}\right),
$$

where $\Delta_{g}$ is the negative Laplacian of $g$ and $R i c_{g}$ is its Ricci tensor. The formal $L^{2}$-adjoint $\mathcal{L}_{g}^{*}$ of the linearized scalar curvature operator $\mathcal{L}_{g}$ is defined as

$$
\mathcal{L}_{g}^{*}(\lambda)=-\left(\Delta_{g} \lambda\right) g+\text { Hess }_{g} \lambda-\lambda \text { Ric }_{g},
$$

where $\operatorname{Hess}_{g} \lambda(X, Y)=g\left(\nabla_{X} D \lambda, Y\right)$ for all vector fields $X, Y$ on $M^{n}$. Throughout the paper, we refer the equation

$$
\mathcal{L}_{g}^{*}(\lambda)=-\left(\Delta_{g} \lambda\right) g+\text { Hess }_{g} \lambda-\lambda \text { Ric }_{g}=0
$$

as Fischer-Marsden equation and a $\lambda$ satisfying this equation as Fischer-Marsden solution. In [7], FischerMarsden conjectured that:

A compact Riemannian manifold $\left(M^{n}, g\right)$ that admits a non-trivial solution of the equation $\mathcal{L}_{g}^{*}(\lambda)=0$ is necessarily an Einstein manifold.

The Riemannian metric $g$ satisfying the equation (1.1) must have constant scalar curvature ( [3] and [7], p.481). In [12], Shen proved that if a 3-dimensional closed manifold $(M, g)$ with positive scalar curvature has a non trivial solution to the equation $\mathcal{L}_{g}^{*}(\lambda)=0$ then $M$ contains a totally geodesic 2 -sphere. It is interesting to note that in [6], Corvino proved $\lambda$ is a nontrivial solution of $\mathcal{L}_{g}^{*}(\lambda)=0$ if and only if the warped product metric $g^{*}=g-\lambda^{2} d t^{2}$ is Einstein. Further, in [3] and [7] it is proved that "If a complete Riemannian manifold $(M, g)$ has a non-trivial solution $\lambda$ of the equation $\mathcal{L}_{g}^{*}(\lambda)=0$, then the scalar curvature $r$ of $g$ is constant". Moreover, the authors Patra-Ghosh studied contact metrics that satisfy Miao-Tam critical condition [14]. Recently, Y. Wang [16] studied the gradient Ricci solitons on $(\kappa, \mu)$-almost CoKähler manifolds and proved that there exist no gradient Ricci solitons on a $(\kappa, \mu)$-almost CoKähler manifold of dimension greater than 3 with $\kappa<0$. 
In particular, Patra-Ghosh [22] proved that if a $K$-contact metric satisfy the Fischer-Marsden equation then it is Einstein. This result intrigues us to consider the Fischer-Marsden equation to some other class of almost contact manifolds. So, we consider the Fischer-Marsden equation on almost CoKähler manifold. The organization of the paper is as follows. In section 2, we recall some basic definitions and formulas. In section 3 , we prove that the Fischer-Marsden equation has only the trivial solution within the framework of an almost CoKähler manifold whose Reeb vector field $\xi$ belongs to the $(\kappa, \mu)$-nullity distribution with $\kappa<0$.

\section{Almost CoKähler manifold}

In this section, we recall some basic definitions and formulas on almost CoKähler manifold which we shall use to prove our results. A $(2 n+1)$ dimensional smooth manifold $M^{2 n+1}$ is said to be an almost contact manifold if it admits a $(1,1)$-type tensor field $\varphi$, a unit vector field $\xi$ (called the Reeb vector field) and a 1-form $\eta$ such that

$$
\varphi^{2} X=-X+\eta(X) \xi, \quad \eta(\xi)=1,
$$

for any vector field $X$ on $M^{2 n+1}$. From (2.1) it is easy to verify that

$$
\varphi(\xi)=0, \quad \eta \circ \varphi=0 .
$$

A Riemannian metric $g$ is said to be an associated (or compatible) metric if it satisfies

$$
g(\varphi X, \varphi Y)=g(X, Y)-\eta(X) \eta(Y),
$$

for all vector fields $X, Y$ on $M^{2 n+1}$. A Riemannian manifold $M^{2 n+1}$ together with an almost contact metric structure $(\varphi, \xi, \eta, g)$ is said to be an almost contact metric manifold. For such a manifold, we can always define a fundamental 2-form $\Phi$ by $\Phi(X, Y)=g(X, \varphi Y)$ for all vector fields $X, Y$ on $M^{2 n+1}$. An almost contact metric manifold is said to be an almost CoKähler manifold if both 1-form $\eta$ and 2-form $\Phi$ are closed. An almost contact metric structure on $M^{2 n+1}$ is said to be normal if the almost complex structure $J$ on $M \times \mathbb{R}$ defined by (e.g., see Blair [2], p.80)

$$
J(X, f d / d t)=(\varphi X-f \xi, \eta(X) d / d t),
$$

where $f$ is a real function on $M \times R$, is integrable. If in addition the almost contact structure is normal, we say that $M^{2 n+1}$ is a CoKähler manifold. An almost contact metric manifold $M^{2 n+1}(\varphi, \xi, \eta, g)$ is CoKähler if and only if $\nabla \varphi=0$ or, equivalently, $\nabla \Phi=0$.

Let $M^{2 n+1}(\varphi, \xi, \eta, g)$ be an almost CoKähler manifold. We now define two operators $h$ and $l$ by $h=\frac{1}{2} £_{\xi} \varphi$ and $l=R(., \xi) \xi$ on $M^{2 n+1}$, where $R$ denotes the curvature tensor and $£$ is the Lie differentiation. These two symmetric tensors of type $(1,1)$ satisfy (e.g.,[17],[18],[19])

$$
h \xi=h^{\prime} \xi=0, \operatorname{Tr} h=\operatorname{Tr} h^{\prime}=0, \quad h \varphi=-\varphi h,
$$

where $h^{\prime}=h \circ \varphi$. On an almost CoKähler manifold the following formulas are valid (See [17],[18],[19]):

$$
\begin{gathered}
\nabla_{X} \xi=h^{\prime} X=h \varphi X, \\
\varphi l \varphi-l=2 h^{2},
\end{gathered}
$$

for any vector field $X$ on $M^{2 n+1}$; where $h^{\prime}=h \circ \varphi$ and $l=R(.,.) \xi$.

A $(\kappa, \mu)$-contact metric manifold is a generalization of Sasakian and $K$-contact manifold. In [4], BlairKoufogiorgos-Papantoniou introduced and studied the notion of $(\kappa, \mu)$-nullity distribution on contact metric manifolds $M^{2 n+1}(\varphi, \xi, \eta, g)$. A contact metric manifold $M^{2 n+1}$ whose curvature tensor satisfies

$$
R(X, Y) \xi=\kappa\{\eta(Y) X-\eta(X) Y\}+\mu\{\eta(Y) h X-\eta(X) h Y\},
$$

for all vector fields $X, Y$ on $M^{2 n+1}$, where $h=\frac{1}{2} £_{\xi} \varphi(£$ denotes the Lie differentiation of $\varphi$ along $\xi$ ) and $\kappa, \mu$ $\in R$ is known as $(\kappa, \mu)$-contact manifold. Several authors study the $(\kappa, \mu)$-contact metric manifold and obtain some interesting results (e.g., [15], [14]). On the other way, we say that $\xi$ belongs to $(\kappa, \mu)$-nullity distribution if the equation (2.6) hold. When $\kappa, \mu$ are smooth functions, it is said that $\xi$ belongs to the generalized $(\kappa, \mu)$-nullity distribution. 


\section{Main Results}

In this section, we study the Fischer-Marsden equation on an almost CoKähler manifold of dimension greater than 3 with $\kappa<0$. First of all, we have the following

An almost CoKähler manifold $M^{2 n+1}(\varphi, \xi, \eta, g)$ is said to be a $(\kappa, \mu)$-almost CoKähler manifold if $\xi$ belongs to the generalized $(\kappa, \mu)$-nullity distribution, i.e., $\xi$ satisfies

$$
R(X, Y) \xi=\kappa\{\eta(Y) X-\eta(X) Y\}+\mu\{\eta(Y) h X-\eta(X) h Y\},
$$

for vector fields $X, Y$ on $M^{2 n+1}$ and $\kappa, \mu$ are smooth functions on $M^{2 n+1}$. A proper $(\kappa, \mu)$-almost CoKähler manifold is a $(\kappa, \mu)$-almost CoKähler manifold with $\kappa<0$. For more details, we refer the reader to [20, 18]. The equation (3.1) gives that $l=-\kappa \varphi^{2}+\mu h$. In view of this, the Eq. (2.5) reduces to

$$
h^{2}=\kappa \varphi^{2} .
$$

To prove our results we require the following lemma.

Lemma 3.1. (Lemma 4.1 of [16]) Let $M^{2 n+1}(\varphi, \xi, \eta, g)$ be a $(\kappa, \mu)$-almost CoKähler manifold of dimension greater than 3 with $\kappa<0$. Then the Ricci operator $Q$ of $M^{2 n+1}$ is given by

$$
Q=\mu h+2 n \kappa \eta \otimes \xi
$$

where $\kappa$ is a non-zero constant and $\mu$ is a smooth function satisfying $d \mu \wedge \eta=0$. Moreover, the scalar curvature of $M^{2 n+1}$ is $2 n \kappa$.

Next, by using the Ricci operator shown by the above lemma, we obtain the following result to prove our main theorem.

Lemma 3.2. Let $M^{2 n+1}(\varphi, \xi, \eta, g)$ be an almost CoKähler manifold of dimension greater than 3 with $\xi$ belonging to the $(\kappa, \mu)$-nullity distribution and $\kappa<0$. Then we have

$$
\left(\nabla_{X} Q\right) \xi=2 n \kappa h^{\prime} X-Q h^{\prime} X
$$

for any vector field $X$ on $M^{2 n+1}$.

Proof: Replacing $\xi$ by $X$ in (3.3) yields $Q \xi=2 n \kappa \xi$. Taking covariant derivative of this along an arbitrary vector field $X$ on $M^{2 n+1}$ we have

$$
\left(\nabla_{X} Q\right) \xi+Q\left(\nabla_{X} \xi\right)=2 n \kappa \nabla_{X} \xi
$$

Making use of (2.4) we complete the proof.

Now, we deduce the expression of the curvature tensor which will help to prove of our main theorem in this section.

Lemma 3.3. Let $(g, \lambda)$ be a non-trivial solution of the Fischer-Marsden equation

$$
-\left(\Delta_{g} \lambda\right) g+H e s s_{g} \lambda-\lambda \operatorname{Ric}_{g}=0
$$

on an almost CoKähler manifold $M^{2 n+1}(\varphi, \xi, \eta, g)$. Then the curvature tensor $R$ on $M^{2 n+1}$ can be expressed as

$$
\begin{aligned}
R(X, Y) D \lambda & =(X \lambda) Q Y-(Y \lambda) Q X+\lambda\left\{\left(\nabla_{X} Q\right) Y-\left(\nabla_{Y} Q\right) X\right\} \\
& -\kappa\{(X \lambda) Y-(Y \lambda) X\},
\end{aligned}
$$

for all vector fields $X, Y$ on $M^{2 n+1}$, where $D$ is the gradient operator with respect to $g$.

Proof: Tracing the equation (3.5) and then using the value of scalar curvature (from lemma 3.1) we obtain $\triangle_{g} \lambda=-\kappa \lambda$. Thus, equation (3.5) can be exhibited as

$$
\nabla_{X} D \lambda=\lambda\{Q X-\kappa X\},
$$


for any vector field $X$ on $M^{2 n+1}$. Taking covariant differentiation of (3.7) along an arbitrary vector field $Y$ on $M^{2 n+1}$, we obtain

$$
\begin{aligned}
\nabla_{Y}\left(\nabla_{X} D \lambda\right) & =(Y \lambda) Q X+\lambda\left\{\left(\nabla_{Y} Q\right) X+Q\left(\nabla_{Y} X\right)\right\} \\
& -\kappa\left\{(Y \lambda) X+\lambda \nabla_{Y} X\right\}
\end{aligned}
$$

for any vector field $X$ on $M^{2 n+1}$. Applying the last equation and (3.7) in the well known expression of the curvature tensor $R(X, Y)=\left[\nabla_{X}, \nabla_{Y}\right]-\nabla_{[X, Y]}$, we complete the proof.

Finally, we may state our main theorem and present the detailed proof as follows.

Theorem 3.1. The Fischer-Marsden equation has only the trivial solution on almost CoKähler manifold of dimension greater than 3 with $\xi$ belonging to the $(\kappa, \mu)$-nullity distribution and $\kappa<0$.

Proof: Taking scalar product of the equation (3.6) with $\xi$ and recalling $Q \xi=2 n \kappa \xi$ we have

$$
\begin{gathered}
g(R(X, Y) D \lambda, \xi)=(2 n-1) \kappa\{(X \lambda) \eta(Y)-(Y \lambda) \eta(X)\} \\
+\lambda\left\{g\left(Y,\left(\nabla_{X} Q\right) \xi\right)-g\left(X,\left(\nabla_{Y} Q\right) \xi\right)\right\} .
\end{gathered}
$$

Making use of (2.3), (3.4), the last equation reduces to

$$
\begin{gathered}
g(R(X, Y) D \lambda, \xi)=(2 n-1) \kappa\{(X \lambda) \eta(Y)-(Y \lambda) \eta(X)\} \\
+\lambda\left\{g\left(X, Q h^{\prime} Y\right)-g\left(Q h^{\prime} X, Y\right)\right\},
\end{gathered}
$$

for all vector fields $X, Y$ on $M^{2 n+1}$. Moreover, setting $X=\varphi X$ and $Y=\varphi Y$ in the last Eq. and noting that $g(R(\varphi X, \varphi Y) D \lambda, \xi)=0$ (it follows from (3.1)) and $h \varphi=-\varphi h$ we have

$$
\lambda\left\{g\left(\varphi X, Q h^{\prime} \varphi Y\right)-g\left(Q h^{\prime} \varphi X, \varphi Y\right)\right\}=0,
$$

for all vector fields $X, Y$ on $M^{2 n+1}$. Making use of (3.3) and $h \varphi=-\varphi h$ we have $\lambda \mu h^{2} \varphi^{2} X=0$ for any vector field $X$ on $M^{2 n+1}$. By virtue of (2.1) and (3.2), the last equation gives $\kappa^{2} \lambda \mu \varphi^{2} X=0$ for any vector field $X$ on $M^{2 n+1}$. Since $\kappa<0$, we have $\lambda \mu=0$. Thus, we have either $\mu \neq 0$, or $\mu=0$. First case gives the trivial solution. Therefore, for later case, we substitute $X$ by $\xi$ in (3.8) and then making use of (2.2), (2.3), $Q \xi=2 n \kappa \xi$ yields

$$
g(R(\xi, Y) D \lambda, \xi)=(2 n-1) \kappa\{(\xi \lambda) \eta(Y)-(Y \lambda)\},
$$

for any vector field $X$ on $M^{2 n+1}$. Setting $X=\xi$ in the Eq. (3.1) and then taking scalar product of the resulting Eq. with $D \lambda$ and using $h \xi=0$ gives

$$
g(R(\xi, Y) D \lambda, \xi)=\kappa g(D \lambda-(\xi \lambda) \xi, Y)+\mu g(D \lambda, h Y),
$$

for any vector field $X$ on $M^{2 n+1}$. Combining last two equations we have

$$
2 n \kappa(D \lambda-(\xi \lambda) \xi)+\mu h D \lambda=0 .
$$

In this case $\mu=0$, so the last Eq. gives $\kappa(D \lambda-(\xi \lambda) \xi)=0$. As $\kappa<0$, so from preceding Eq. we infer that $D \lambda=(\xi \lambda) \xi$. Taking covariant differentiation of the last Eq. along an arbitrary vector field $X$ on $M^{2 n+1}$ together with (2.1), (2.4) entails that

$$
\nabla_{X} D \lambda=X(\xi \lambda) \xi+(\xi \lambda) h^{\prime} X
$$

Making use of this in the Eq. (3.7), we can find

$$
\lambda Q X=X(\xi \lambda) \xi+(\xi \lambda) h^{\prime} X+\kappa \lambda X,
$$

for any vector field $X$ on $M^{2 n+1}$. Comparing the value of $Q X$ from relation (3.3) and the last Eq., we compute

$$
\kappa \lambda X+(\xi \lambda) h^{\prime} X-\lambda \mu h X-2 n \kappa \lambda \eta(X) \xi+X(\xi \lambda) \xi=0,
$$

for any vector field $X$ on $M^{2 n+1}$. Tracing this over $X$ we have

$$
(2 n+1) \kappa \lambda-2 n \kappa \lambda+\xi(\xi \lambda)=0 .
$$


Substituting $X$ by $\xi$ in (3.7) and then taking scalar product with $\xi$ yields $\xi(\xi \lambda)=2 n \kappa \lambda-\kappa \lambda$, which together with the Eq. (3.9) gives $2 n \kappa \lambda=0$. As $\kappa<0$, from last Eq. we infer that $\lambda=0$. This completes the proof.

Note that if we consider $\kappa$ and $\mu$ as smooth functions instead of constants then, the lemma 3.1 is true for manifold $M^{2 n+1}$ of dimension greater than or equal to 3 (see [20]) and therefore the scalar curvature of $M^{2 n+1}$ is $2 n \kappa$ (from lemma 3.1). Since the metric $g$ satisfying the equation (1.1) must have the constant scalar curvature (see [3] and [7], p.481), we can conclude $\kappa$ is constant. Hence the lemma 3.2 is also applicable. Then, from theorem 3.1 we have

Corollary 3.1. Let $M^{2 n+1}(\varphi, \xi, \eta, g), n>1$, be a $(\kappa, \mu)$-almost CoKähler manifold with $\kappa<0$. Then the FischerMarsden equation has only the trivial solution on $M^{2 n+1}$.

Proof: Proceeding same as theorem 3.1, we have $\lambda \mu=0$. Suppose $\lambda \neq 0$ in some open set $\mathcal{O}$ of $M^{2 n+1}$. Then $\mu=0$ on $\mathcal{O}$. Following the proof of the above theorem we conclude that $\lambda=0$ on $\mathcal{O}$. Hence $\lambda=0$ on $M^{2 n+1}$. This completes the proof.

\section{Acknowledgments}

The author D. S. Patra is financially supported by the Council of Scientific and Industrial Research, India (grant no. 17-06/2012(i)EU-V).

\section{References}

[1] Besse, A., Einstein manifolds. Springer-Verlag, New York, 2008.

[2] Blair, D. E., Riemannian geometry of contact and symplectic manifolds. Birkhauser, Boston, 2002.

[3] Bourguignon, J. P., Une stratifcation de l'espace des structures riemanniennes. Compositio Math., 30 (1975), 1-41.

[4] Blair, D. E., Koufogiorgos, T. and Papantoniou, B. J., Contact metric manifolds satisfying a nullity condition. Israel J. of Math., 91 (1995), 189-214.

[5] Cernea, P. and Guan, D., Killing fields generated by multiple solutions to the Fischer-Marsden equation. International Journal of Math., 26 (2015), 93-111.

[6] Corvino. J., Scalar curvature deformations and a gluing construction for the Einstein constraint equations. Commun. Math. Phys., 214 (2000), 137-189.

[7] Fisher A. and Marsden. J., Manifolds of Riemannian metrics with prescribed scalar curvature. Bull. Am. Math. Soc., 80 (1974), $479-484$.

[8] Kobayashi, O., A Differential Equation Arising From Scalar Curvature Function. J. Math. Soc. Japan, 34 (1982), 665-675.

[9] S. B. Myers: Connections between differential geometry and topology. Duke Math. J., 1 (1935), 376-391.

[10] Obata, O., Certain conditions for a Riemannian manifold to be isometric with a sphere. J. Math. Soc. Japan, 14 (1962), no. 3, 333-340.

[11] Olszak, Z., On contact metric manifolds. Tohoku Math. J., 31 (1979), 247-253.

[12] Shen, Y., A note on Fischer-Marsden's conjecture. Proc. Am. Math. Soc., 125 (1997), 901-905.

[13] Tanno, S., The topology of contact Riemannian manifolds. Illinois J. Math., 12 (1968), 700-717.

[14] Patra, D. S. and Ghosh, A., Certain contact metrics satisfying Miao-Tam critical condition. Ann. Polon. Math., 116 (2016), no. 3, $263-271$.

[15] Sharma, R., Certain results on $K$-contact and $(k, \mu)$-contact manifolds. J. Geom., 89 (2008), no. (1-2), 138-147.

[16] Wang, Y., A Generalization of the Goldberg Conjecture for CoKähler Manifolds. Mediterr. J. Math., 13 (2016), $2679-2690$.

[17] Chinea, D., Léon, M. de and Marrero, J. C., Topology of cosymplectic manifolds. J. Math. Pures Appl., 72 (1993), no. 6, 567-591.

[18] Marrero, J. C. and Padŕon, E., New examples of compact cosymplectic solvmanifolds. Arch. Math. (Brno), 34 (1998), no. 3, $337-345$.

[19] Dacko, P. and Olszak, Z., On almost cosymplectic $(k, \mu, \nu)$-space. Banach Center Publ., 69 (2005), 211-220.

[20] Endo, H., Non-existence of almost cosymplectic manifolds satisfying a certain condition. Tensor (N. S.), 63 (2002), no. 3, 272-284

[21] Gray, A., Spaces of contancy of curvature operators. Proc. Amer. Math. Soc. 17 (1966), 897-902.

[22] Patra, D. S. and Ghosh, A., Fischer-Marsden conjecture and contact geometry. comunicated.

\section{Affiliations}

DHRITI SUNDAR PATRA

ADDRESS: Jadavpur University, Dept. of Mathematics, 700 032, Kolkata-India.

E-MAIL: dhritimath@gmail.com 
ARINDAM BHATTACHARYYA

AdDRESS: Jadavpur University, Dept. of Mathematics, 700 032, Kolkata-India.

E-MAIL: bhattachar1968@yahoo.co.in

MANJUSHA TARAFDAR

AdDRESS: University of Calcutta, Dept. of Pure Mathematics, 700019, Kolkata-India.

E-MAIL: drmanjushamajumdar@gmail.com 